\title{
enCOMPASS - an Integrative Approach to Behavioural Change for Energy Saving
}

\author{
Piero Fraternali*, Sergio Herrera*, Jasminko Novak ${ }^{\ddagger}{ }^{\ddagger}$, Mark Melenhorst ${ }^{\ddagger}$, Dimitrios Tzovaras ${ }^{\dagger}$, Stelios Krinidis ${ }^{\dagger}$, \\ Andrea Emilio Rizzoli ${ }^{\S}$, Cristina Rottondi ${ }^{\S}$ and Francesca Cellina ${ }^{\S}$ \\ * Department of Electronics, Information, and Bioengineering, Politecnico di Milano, Italy \\ \{piero.fraternali, sergioluis.herrera\}@ polimi.it \\ ${ }^{\dagger}$ Information Technologies Institute, Centre for Research and Technology Hellas, Greece \\ \{dimitrios.tzovaras, krinidis\}@iti.gr \\ $\ddagger$ European Institute for Participatory Media, Berlin - \{j.novak, m.melenhorst $\} @$ eipcm.org \\ $\S$ Dalle Molle Institute for Artificial Intelligence (IDSIA) - University of Lugano (USI) - \\ University of Applied Science and Arts of Southern Switzerland (SUPSI) - \{name.surname\}@supsi.ch \\ ฯ University of Applied Sciences Stralsund, Germany - jasminko.novak@fh-stralsund.de
}

\begin{abstract}
This paper presents the research objectives of the enCOMPASS project, which aims at implementing and validating an integrated socio-technical approach to behavioural change for energy saving. To this end, innovative user-friendly digital tools will be developed to 1) make energy data consumption available and understandable for different types of users and stakeholders (household residents, office employees, school pupils, building managers, utilities, ICT providers) and to 2) empower them to collaborate in order to achieve energy savings and manage their energy needs in efficient, cost-effective and comfort-preserving ways. The project will demonstrate how this can be achieved with a novel approach that integrates user-centered visualisation of energy data from smart sensors and user-generated information with context-aware collaborative recommendations for energy saving, intelligent control and adaptive gamified incentives enabling effective and sustained behavioural change.
\end{abstract}

Index Terms-Smart Metering; Sensors; Behavioral Change; Serious Games; Gamification;

\section{INTRODUCTION}

The European energy strategy for 2030 targets a $40 \%$ reduction in $\mathrm{CO}_{2}$ emissions compared to 1990 levels and a $27 \%$ energy saving respect to the business-as-usual scenario [1]. Achieving such goals requires major efforts from citizens, companies, and policy makers alike. It has been recognized that structural policy measures (e.g. subsidising energy efficient building renovation) and technological progress alone (e.g. smart meters, smart home technology), even though important, do not suffice. Without effective solutions to manage energy demand by stimulating energy savings through behavioural change of consumers, the above mentioned targets cannot be reached. The combination of persuasive technologies and IoT technologies (e.g. smart metering, home automation, sensing and mobile devices) are a powerful enabler to facilitate this behavioural change. Existing experiences with persuasive technologies suggest that effective designs should incorporate different types of feedback and analysis options (e.g. offering possibility to analyze historical series of consumption data or to make social comparisons), accompanied by suitable motivational techniques (e.g. individual, social and economical rewards) and energy saving suggestions [2]. But to be effective, such feedback, motivational triggers and suggestions for energy saving actions must consider the specific characteristics of different consumer types and different individual needs (e.g comfort levels), and must be presented in a timely manner and at the right moment to attract attention. They need to be adapted to a specific situation in order to provide actionable suggestions tailored to a given user and her current activity context [3].

While single aspects are being researched, such integrated, context-aware, adaptive and user-tailored solutions are still missing. Individual solutions to collect energy consumption data from smart meters and smart home devices exist, but making energy consumption data available to consumers in easyto-use, easy-to-understand ways, so that they can stimulate behaviour change, is still a challenge. Existing tools provide partial data from individual sources not linked to each other and the provided energy saving tips tend to be general, in a "one size fits all" manner [4]. Metering services, smart home sensors and existing consumer tools are not integrated with each other, due to often different, non-cooperative providers. Moreover, typical solutions do not address the full cycle of behavioural change and thus cannot harness the full potential of demand management. To induce behavioural change and sustain it in the long term, overcoming barriers such as future discounting or rebound effects (e.g. by providing small but immediate rewards rather than large ones later on, activating social norms by propagating socially-desirable actions), socio-technical systems are actually required that incorporate insights from behavioural sciences and environmental psychology. For effective and sustained behavioural change, all of these individual enablers, mechanisms and techniques need to be integrated in a holistic socio-technical system, capable of aligning technological enablers with state-of-the-art models of behavioural change processes. Such integrated socio-technical systems for energy saving and behavioural change have been theorized in research, but are not yet available in practice nor have been validated in real-world pilots. In the remainder 
of the paper, first the main enCOMPASS research objectives are laid out in Section II, then a short overview of related literature in Section III supports the project rationale, whereas in Section IV the structure of the proposed enCOMPASS framework is given. The pilots where the system will be deployed and assessed are presented in Section V. Even though the project has just recently started, the presented description of the proposed approach can already inform existing and future work in the field.

\section{RESEARCH OBJECTIVES}

In order to target the above mentioned shortcomings of the current approaches, the enCOMPASS - Collaborative Recommendations, Visualisation and Adaptive Control for Personalised Energy Saving - project targets the following main objectives:

- Stimulate behavioural change for energy saving with a holistic approach integrating innovative digital tools with smart home automation and a full-cycle model of sustained behavioural change.

- Visualize energy consumption data for consumers in a user-friendly, easy-to-understand way that translates the abstract, numeric consumption data into a semantically understandable format for the users.

- Demonstrate that individual comfort levels can be maintained while achieving energy savings. Recommending targeted energy saving actions to users, based on their comfort profile, current context, activity, and best-fitting energy control profiles, will allow users to save energy while retaining personal comfort levels.

- Validate and compare the effectiveness of the different types of behavioural change interventions (visualisation, adaptive gamified incentives, context-based recommendations and control) in three different types of buildings and settings (residential, schools, public buildings) for different user types (households, school classes, office employees, visitors), in three different cultural and climatic conditions (Germany, Greece and Switzerland).

The enCOMPASS project is supported by the European Commission since November 2016 under the Horizon 2020 funding program. The project is coordinated by Politecnico di Milano and brings together fourteen European partners from seven countries: European Institute for Participatory Media, Stadtwerk Hafurt, Naturschutzbund Deutschland - NABU (Germany), Watt and Volt Anonimi Etairia Ekmetalleysis Enallaktikon Morfon Energeias, Ethniko Kentro Erevnas Kai Technologikis Anaptyxis, Ethniko Idryma Erevnon (Greece), Societ Elettrica Sopracenerina, Scuola Universitaria Professionale della Svizzera Italiana - SUPSI, Paradox Engineering (Switzerland), Set Mobile (Romania), Kaleidospublishing (Italy), Kauno Technologijos Universitetas (Lithuania) e Gravity Research \& Development Kutato (Hungary).

\section{RELATED WORK}

Energy consumption feedback holds the promise of reducing energy consumption with levels by $4 \%-12 \%$ on average, with peak savings going beyond $20 \%$ [2]. Some EU projects (e.g.
GreenPlay [5], EnerGAware [6]) even aim at achieving up to $30 \%$, although these claims yet need to be demonstrated - and they do not aim at preserving user comfort levels. The deployment of smart meters in an increasing number of households has given a boost to research attention for behavioural change based on energy consumption feedback. Different energy saving applications embedded in users' everyday environment have been researched [7], [8], using visualized consumption feedback and gamified social interactions to motivate people to adopt energy-efficient lifestyles. Many are data-oriented (e.g. bar or pie charts of consumption [9]), some are closely connected to the real consumption context (e.g. floor plans [9]), others metaphorical (e.g. traffic lights and gauges [9]), playful and ambient (BeAware [10], [11]), or connected to nature or animal habitats (eco-visualization [12], [13]). They have shown various levels of success and intelligibility for the users. To visualize consumption effectively and facilitate long-term sustainable behaviour, consumption should be broken down, e.g. temporally, by events, per appliance or type of consumption [12]-[14]. Benchmarks for comparison are especially important, to better understand whether consumption is "normal", "excessive", or "economical". Different comparisons can be shown, e.g. historical for self-comparison, normative for comparison with other households or individuals and "social" for comparison against others "in their collective social setting", e.g. individuals in the same household [15] or neighborhood [11]. But stimulating behaviour change effectively requires visualization that is easily understandable at a glance and seamlessly embedded into user's context offering details and comparisons based on the current activity situation, and relating it to possible energy saving actions. Closing this loop effectively in a user-friendly way is still a challenge. Consumption tips and advices are a common strategy to motivate energy saving [11], [14], [16], [17], sometimes provided in a contextualized but not in a personalized manner [14], [17], and rarely shown in relation to direct consumption visualisation.

A new direction in addition to the display of consumption feedback is the use of personalized recommendations employing machine learning techniques to identify different consumer classes, create user models and map appropriate action tips in a personalized manner based on user class and current context. While the combination of energy visualization and feedback with recommendations is promising, little evidence is available about the effective combinations of feedback and other measures, nor about the sustainability of the effects on energy consumption over time. The vast majority of energy behaviour research has focused on influencing behaviour determinants (e.g. attitudes, beliefs, and behavioural control) without positioning the interventions in the behavioural change process [18]. But there is growing awareness that interventions and incentives need to be provided based on the stage in the behavioural change process, i.e. that a one-size-fits-all approach does not work [19]. Models such as the transtheoretical model for behavioural change applied to energy consumption show how change in energy behaviour occurs through different phases [2], [19]: from becoming aware of the need to change behaviour, to understanding concrete actions to save energy, to performing them and ultimately developing new (automatic) 
behavioural habits.

However, holistic attempts of supporting this behavioral change model by designing integrated systems that exploit the full potential of ICT and smart energy infrastructures, still need to be undertaken. To this aim, the enCOMPASS model integrates insights from the transtheoretical model of behavioural change [19], its adaptation to pro-environmental behaviour by [20], and its application to smart metering by [2], but goes beyond the state-of-the-art literature: it will not only develop personalized applications based on sensor data, but also adapt feedback and recommendations to the current activity and situational context of the consumer, and link back to automated indoor climate control systems. In this way, both the most suitable incentives and action recommendations can be presented for a given user (type) in a personalised and context-aware manner, maximizing the likeliness of user engagement and behavioural change.

Finally, the goal of enCOMPASS is to enable the achievement of energy saving targets while preserving indoor comfort, a challenge that has barely been addressed by previous research projects.

\section{THE ENCOMPASS FRAMEWORK}

\section{A. Technical Approach}

The holistic socio-technical system that enCOMPASS will implement and validate is depicted in Figure 1: the system will enable an interactive visualisation of energy consumption, allowing the users to explore consumption data in more detail: e.g. by time (day, week, year), by consumption source, by user context and activity class. This will trigger the consumers' individual awareness of their energy consumption and of how they are personally affected, as well as collective awareness of how it affects their environment. The system will explicitly warn users when they are using too much energy and provide advice on how to improve their consumption (recommender for energy saving actions).

Users' motivation to perform recommended actions will be stimulated by two-types of gamification elements: $i$ ) gamified rewards (points, badges, achievements, tangible prizes) and ii) a serious game, combining a physical board-game with a digital app. These will employ different types of gamemechanics such as goal setting (e.g. personal saving goals rewarded by bonus points and real rewards such as vouchers), social comparison and social collaboration (e.g. collecting points in teams for performing energy saving actions, competing with others). Such gamified incentives will be provided in an adaptive way, considering different user types and their behaviour in using the system (e.g. pushing triggers suited for a given user through contextualised notifications).

Context-based, collaborative recommendations of energy saving actions will include recommendations of similar users, whose (anonymised) energy usage profiles and actions will be displayed to the user. In this way, collaboration between users will be stimulated, allowing the users to learn from others how to optimize energy consumption. By analyzing user consumption patterns and his/her activity profile (e.g. time slots of room occupancy), the system will compute user profiles and recommend control actions for the smart home devices. To increase effectiveness, whenever possible automated recommended actions confirmed by a user will be automatically implemented by the enCOMPASS system, through its connection to the in-door control system (e.g. adjusting heating temperature, turning off lights in unoccupied rooms). Context detection and analysis will combine consumption data from smart metering and smart home devices with privacy-preserving detection of user presence and activity in different areas of the household based on mobile $\mathrm{WiFi}$ sensing. In doing so, the system will consider appropriate comfort levels for a given user and context, and integrate this into the energy saving recommendations and visualisations. To increase the precision of comfort detection and contextbased recommendations, the system will employ a "humanin-the-loop" technique, allowing users to manually validate system results (e.g. changing the comfort level) and improve the learning algorithms.

\section{B. Infrastructure Deployment}

In architectural terms, the enCOMPASS system is conceived as an open, extensible, process-based, cloud-enabled platform, combining $i$ ) energy usage information from inhome sensors (smart meters and communication-enabled smart home appliances for heat and electricity), $i i$ ) user-generated information (automatic and manual activity tracking), iii) adaptive gamified energy visualisation, and $i v$ ) intelligent controls and automation to achieve sustainable changes in user energy consumption patterns and without compromising the users comfort level.

This architecture will be deployed in a cloud infrastructure to allow for easy application development by designated third-parties, following the platform-as-a-service (PaaS) and software-as-a-service (SaaS) models. To maximize impact in real-world scenarios, the enCOMPASS system will be easily deployable, easily configurable and customizable to different settings and business scenarios (e.g. different utilities working with different technology providers). It will provide open interfaces to energy data and business services, stimulating market uptake and business development through open innovation and new kinds of white-label solution, thus initiating the creation of a business ecosystem for the development and provision of value-added services for smart energy demand management.

The core enCOMPASS components are:

Sensors data acquisition: including energy related data, stemming from smart electricity meters and smart plugs at individual appliance level, and contextual data, such as environmental data to cover comfort related data. The smart electricity meters internally store the energy consumption data measured at fine resolution (e.g. every 15 or 60 minutes), which are transmitted on daily basis to the concentrator located at the nearly transformer station (via either power line communication or wireless neighborhood area networks). The data are then sent to the utilities' servers and subsequently processed and exported as an xml file on the project FTP server. Similarly, sensors data are transmitted via the wireless home (or neighborhood) area network and gathered by a 


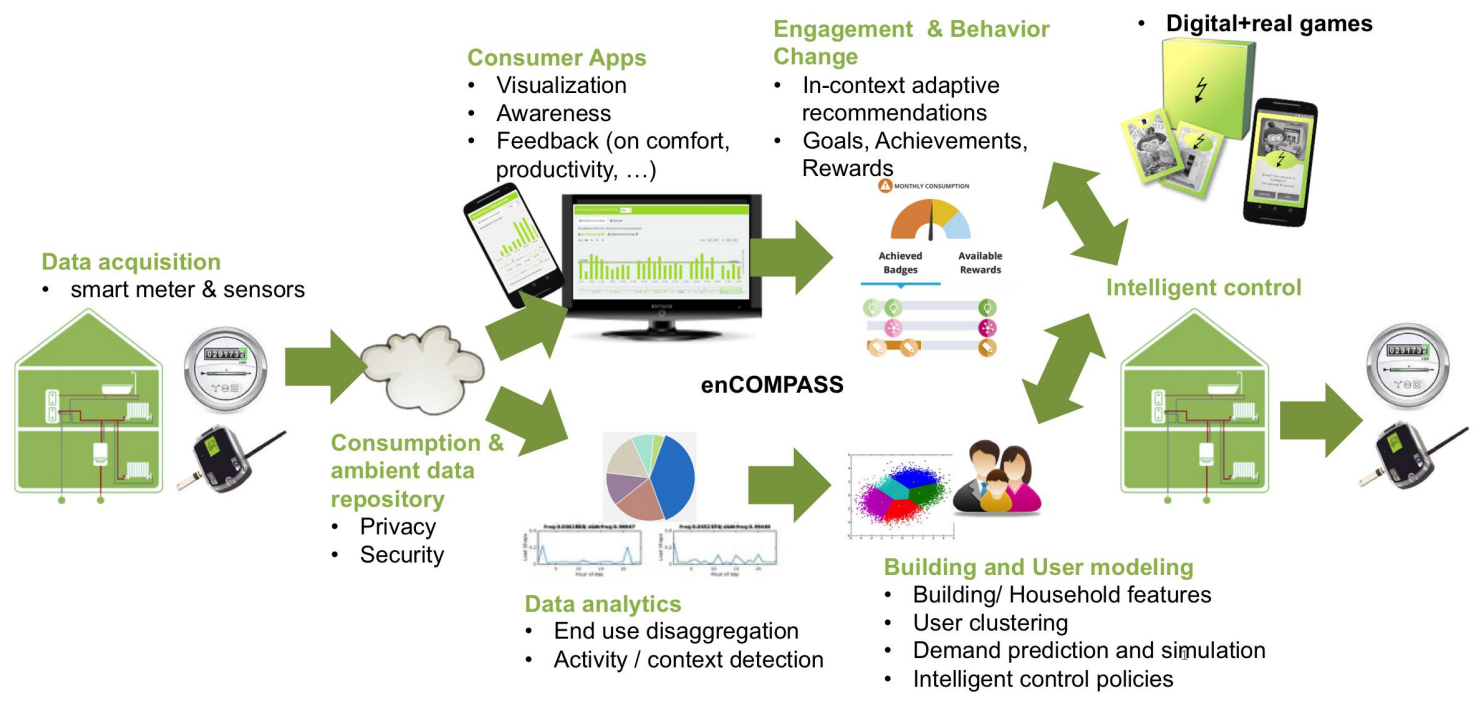

Fig. 1. The EnCOMPASS general framework

local concentrator (either at home or neighborhood level), which conveys them to the utilities' servers via standard wired/wireless connections.

User data acquisition: including psychographic variables acquisition from gamified mobile apps, instant poll data acquisition (e.g., quick feedback on activity, comfort conditions), along with data inferred in a privacy preserving way, through activity data produced by users' smartphones (such as e.g. WiFi or Bluetooth signals).

Data analysis and user modeling: algorithms for extracting activity from sensor and app data, profiling different types of user behaviour, inferring activity context, predicting reactions to stimuli (e.g., saving tips).

Data analysis and building modelling: algorithms for estimating the comfort levels and energy behaviour of buildings based on a reduced set of measured input parameters dependent on the class of the buildings. They allow projecting the impact of consumers' actions on energy consumption and on the status of the building, to complete the user profile. The base model includes: thermal building characteristics (i.e., envelope materials, building size), user profile, local climate data, energy consumption of subsystems (i.e., HVAC, plug loads etc.).

Adaptive in-context action recommendation: used in order to map user's consumption, models and behavioural data into personalized recommendations for energy saving. Users will be classified in categories and activity patterns will be extracted from consumption, sensors and psychographic data in a privacy respecting way, in order to compute energy saving recommendations for a specific user category.

Engagement engine with adaptive gamification: a rulebased engine that maps actions into achievements and assigns rewards. Actions are abstract occurrences that can be associated to a variety of sources, such as building sensors (e.g. switching off an appliance, reaching a set temperature, saving a set amount of energy), and users (execution of a recommended action, in-game actions, manual data input, etc.). Rewards can be immaterial (scores, badges) or material (coupons, re- deemable goods). The engine has a plug-in architecture so that actions, achievements, rewards and rules can be customized, without programming, by the building manager.

Semantic data repository (SDR): it stores facts about all the entities managed by the platform, namely: people and their relationships, buildings and their conditions, behavioural clusters and users' segments, actions, achievements, rewards, activities and contexts, recommendations. The SDR also contains people-to-people relations, to denote household, friendship, group membership, endorsement, recommendation, and affinity. People and groups can have multiple in-context profiles, denoting current activity and behavioural state.

Energy efficiency assessment console: it is an advanced interface whereby building managers and utilities can analyse the energy consumption and system effectiveness and set saving goals/incentives.

Behavioural change apps: web and mobile applications developed to visualize energy usage, they deliver recommendations and engage users with serious game mechanics and gamification techniques.

enCOMPASS APIs: they provide on-demand, cloudbased web services that enable end-user applications to access and exploit the enCOMPASS processes. The data and service APIs enable developers to access both enCOMPASS functionalities and data while implementing their own services.

\section{Pilot Deployments}

The effectiveness of the enCOMPASS system will be assessed in three pilots under three different climate zones (humid continental, hot-summer Mediterranean, continental subarctic), three building types (residential buildings, schools, office buildings) and three different cultural settings (central European, Mediterranean, sub-alpine European), in three different European countries (Germany, Greece, Switzerland). Climate differences must be addressed since the perceived necessity of consuming energy can differ according to the climate conditions (e.g. use of air conditioning or heating). Differences between building types yield different saving 
TABLE I

SUMMARY OF PILOT DEPLOYMENTS

\begin{tabular}{|l|l|l|l|}
\hline Pilot Location & Germany - Hassfurt & Greece: Athens and Thessaloniki & Switzerland - Locarno \\
\hline Building types & $\begin{array}{l}\text { Residential, school and pub- } \\
\text { lic buildings }\end{array}$ & $\begin{array}{l}\text { Residential, school (college) and public build- } \\
\text { ings (public library and utility company offices) }\end{array}$ & $\begin{array}{l}\text { Residential, school (primary education) and } \\
\text { public building (municipality building) }\end{array}$ \\
\hline Climate description & Humid continental climate & Hot-summer mediterranean & Continental subarctic \\
\hline Actors involved & $\begin{array}{l}\text { Residents, school students } \\
\text { and staff, public administra- } \\
\text { tion employees }\end{array}$ & $\begin{array}{l}\text { Residents, college students and staff, library staff } \\
\text { and visitors, office personnel and visitors }\end{array}$ & $\begin{array}{l}\text { Residents, school students and staff, public } \\
\text { administration employees and municipality } \\
\text { visitors }\end{array}$ \\
\hline
\end{tabular}

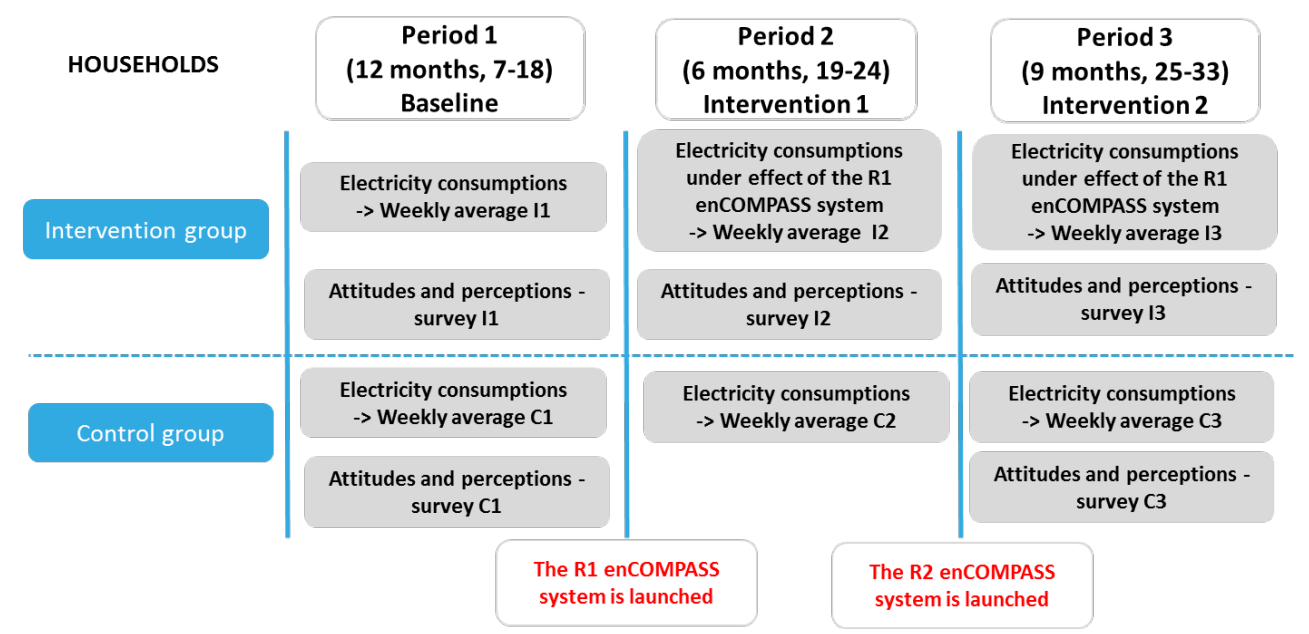

Fig. 2. Timeline of the enCOMPASS experiments

potentials and different beliefs and attitudes towards the energy consumption in the building. Perceived behavioural control (the extent to which a user feels capable of saving energy) also differs between buildings (e.g. a student may not feel capable of influencing consumption of the school building). Finally, differences in cultural factors can influence environmental beliefs and the propensity to save energy. Characteristics of the pilot sites are summarized in Table I. In each pilot, one hundred households, one school and one public building will be actively involved, thus allowing to assess the achieved changes in everyday energy activities and savings for four target groups (residents, public administration employees, school students and staff) in three different settings. Each building will be equipped with one electricity smart meter, a few smart plugs for electric appliances, and four sensors (luminance, presence, indoor/outdoor temperature). Changing behaviour and energy savings due to the use of the enCOMPASS system will be quantitatively assessed in terms of electricity consumption, following the eeMeasure methodology [21]. In addition, user perceptions and attitudes will be assessed, using methods typically employed in user-centered design research and psychological research, such as questionnaires, focus groups and interviews.

Figure 2 reports the general structure of the pilot experiments, referring in particular to households. For residential users, activities envision two sample groups: an Intervention and a Control group. The size of the Control groups for residential users is expected to be the same as the Intervention group (one hundred households). If the number of households equipped with electricity smart meters located in the same area is sufficiently high, the three Control groups will be created by stratified random sampling. More in detail, households of the Intervention groups will be stratified according to the following parameters:

- size: single-person/couple/more than two person households;

- type of house: single-family house (including terraced house)/apartment;

- type of heating: electricity-fed (heat pump, direct electricity)/oil/gas/wood/other;

- type of hot water boiler: electricityfed/oil/gas/wood/other.

The same stratification rules will be applied on all the households located in the pilot areas. Three further samples of one hundred households will then be randomly selected among them, adopting the same proportions as the related Intervention groups, in order to create three Control groups. The Control groups will be thus totally uninfluenced by any measure or action channelled through the enCOMPASS platform: neither they will receive other information on their consumption than the regular energy bills, nor they will be aware of being monitored within enCOMPASS. Regarding schools and public buildings, instead, no control group is envisioned, since finding a school for the control group that is comparable to that of the intervention group (in building type, pupil/employee composition etc.) is virtually impossible in small settings in the German and Swiss pilots as it is for the private college in the Greek pilot, that is unique in the city of Thessaloniki. The eeMeasure methodology is still applicable even in absence of control groups, by applying regression models to estimate the projected energy consumption after the intervention.

Three monitoring periods are envisioned, which run consecutively for eighteen months, and during which electricity consumption (smart meters), user patterns and context charac- 
teristics (sensors) and qualitative data (surveys and interviews) are collected.

1) Monitoring period 1 (Baseline) lasts 12 months. It allows to collect a rich baseline set of data, allowing to take account of variabilities due to the different periods of the year (temperature and duration of the day, which directly influence heating consumptions, but also indirectly influence people permanence at home and the related energy consumptions);

2) Monitoring period 2 (Intervention period 1) lasts 7 months. It allows to assess effectiveness of the first release of the enCOMPASS platform (R1), respect to the baseline period;

3) Monitoring period 3 (Intervention period 2) lasts 9 months and allows to assess effectiveness of the second release of the enCOMPASS platform (R2), again respect to the baseline period.

In periods 2 and 3 only Intervention groups will be using the enCOMPASS platform (respectively, releases R1 and R2). Control groups, instead, will not be influenced by any enCOMPASS tool. Quantitative assessment of the enCOMPASS effectiveness will be performed in each pilot by means of a difference in differences approach [22]: differences in electricity consumptions will be accounted for (Period 2 and Period 1) and (Period 3 and Period 1), both for Intervention and Control group. Such differences in electricity consumptions will then be compared between the Intervention and the Control group. This will allow to univocally attribute the expected positive effects in terms of reduction in electricity consumptions to the enCOMPASS platform.

\section{ONGOING WORK}

The described approach and enCOMPASS architecture are currently being implemented in a user-centered process combining a "technology push" and "user pull". The requirements analysis aligns the technological enablers and project objectives with end-user requirements and constraints in order to ensure user acceptance and produce a detailed system specification guiding the implementation. Participating households are being recruited, the first data sets with historical consumption data have been collected as a basis for calculating the baselines, and the detailed specifications of sensor configurations for the pilots have been produced. The smart metering infrastructure and the technical integration model allowing existing components and smart home apps of the utilities to be integrated with the enCOMPASS system are being completed. In the next steps the full user requirements and system specification will be produced and implemented, followed by a first release of the enCOMPASS platform that will be iteratively extended. Its validation in the pilots will be performed under real-world conditions over a period of eighteen months.

\section{ACKNOWLEDGEMENTS}

This work is partially supported by the "enCOMPASS Collaborative Recommendations and Adaptive Control for Personalised Energy Saving" project funded by the EU H2020 Programme, grant agreement no. 723059.

\section{REFERENCES}

[1] "2030 energy strategy," 2017, accessed: 25 February 2017. [Online]. Available: https://ec.europa.eu/energy/en/topics/energy-strategy/ 2030-energy-strategy

[2] M. Nachreiner, B. Mack, E. Matthies, and K. Tampe-Mai, "An analysis of smart metering information systems: a psychological model of selfregulated behavioural change," Energy research \& social science, vol. 9, pp. 85-97, 2015.

[3] I. Micheel, J. Novak, P. Fraternali, G. Baroffio, A. Castelletti, and A. Rizzoli, "Visualizing \& gamifying water \& energy consumption for behavior change," Proc. Fostering Smart Energy Appl. (FSEA), 2015.

[4] M. Z. Huber and L. M. Hilty, "Gamification and sustainable consumption: overcoming the limitations of persuasive technologies," in ICT Innovations for Sustainability. Springer, 2015, pp. 367-385.

[5] "Greenplay project - game to promote energy efficiency actions," H2020-EU.3.3.1, No. 649621, 2015-18, accessed: 25 February 2017. [Online]. Available: http://greenplay-project.eu/

[6] "Energaware project - energy game for awareness of energy efficiency in social housing communities," H2020-EU.3.3.1, No. 649673, 2015-18, accessed: 25 February 2017. [Online]. Available: http://energaware.eu/

[7] A. Barbato, C. Bolchini, A. Geronazzo, E. Quintarelli, A. Palamarciuc, A. Pit, C. Rottondi, and G. Verticale, "Energy optimization and management of demand response interactions in a smart campus," Energies, vol. 9, no. 6, 2016.

[8] C. Rottondi, M. Duchon, D. Koss, A. Palamarciuc, A. Piti’, G. Verticale, and B. Schaetz, "An energy management service for the smart office," Energies, vol. 8, no. 10, pp. 11667-11684, 2015.

[9] P. Monigatti, M. Apperley, and B. Rogers, "Power and energy visualization for the micro-management of household electricity consumption," in Proc. Conf. Advanced Visual Interfaces. ACM, 2010, pp. 325-328.

[10] "Be aware project - boosting energy awareness with adaptive real-time environments," EU FP7-ICT, No. 224557,, 2008-11, accessed: 25 February 2017. [Online]. Available: http://www.energyawareness.eu/

[11] D. Wemyss, R. Castri, V. De Luca, F. Cellina, V. Frick, E. Lobsiger-Kägi, P. G. Bianchi, C. Hertach, T. Kuehn, and V. Carabias, "Keeping up with the joneses: examining community-level collaborative and competitive game mechanics to enhance household electricity-saving behaviour," in Proceedings of the 4th European Conference on Behaviour and Energy Efficiency Behave 2016, 2016.

[12] A. Gustafsson, M. Bång, and M. Svahn, "Power explorer: a casual game style for encouraging long term behavior change among teenagers," in Proc. Adv. in Comp. Entertainment Tech. ACM, 2009, pp. 182-189.

[13] A. Gustafsson, C. Katzeff, and M. Bang, "Evaluation of a pervasive game for domestic energy engagement among teenagers," Computers in Entertainment (CIE), vol. 7, no. 4, p. 54, 2009.

[14] G. Jacucci, A. Spagnolli, L. Gamberini, A. Chalambalakis, C. Björkskog, M. Bertoncini, C. Torstensson, and P. Monti, "Designing effective feedback of electricity consumption for mobile user interfaces." PsychNology Journal, vol. 7, no. 3, pp. 265-289, 2009.

[15] "3ehouses, saving energy \& environment across europe," accessed: 25 February 2017. [Online]. Available: http://www.3ehouses.eu/

[16] M. Peham, G. Breitfuss, and R. Michalczuk, "The ecogator app: gamification for enhanced energy efficiency in europe," in Proc. Conf. on Tech. Ecosys. for Enhancing Multiculturality. ACM, 2014, pp. 179-183.

[17] L. Gamberini, N. Corradi, L. Zamboni, M. Perotti, C. Cadenazzi, S. Mandressi, G. Jacucci, G. Tusa, A. Spagnolli, C. Björkskog et al., "Saving is fun: designing a persuasive game for power conservation," in Proc. Adv. in Comp. Entertainment Tech. ACM, 2011, p. 16.

[18] E. van der Werff and L. Steg, "One model to predict them all: predicting energy behaviours with the norm activation model," Energy Research \& Social Science, vol. 6, pp. 8-14, 2015.

[19] H. A. He, S. Greenberg, and E. M. Huang, "One size does not fit all: applying the transtheoretical model to energy feedback technology design," in Proc. of ACM CHI'10. ACM, 2010, pp. 927-936.

[20] S. Bamberg, "Changing environmentally harmful behaviors: A stage model of self-regulated behavioral change,' Journal of Environmental Psychology, vol. 34, pp. 151-159, 2013.

[21] G. Lohmann, G. Heilmann, U. Hacke, and S. Robinson, "The ICT PSP methodology for energy saving measurement," 2011, accessed: 25 February 2017. [Online]. Available: http://cordis.europa.eu/docs/projects/cnect/6/250496/ 080/deliverables/001-ARES975520CIPCommondeliverableeSESH.pdf

[22] A. Abadie, "Semiparametric difference-in-differences estimators," The Review of Economic Studies, vol. 72, no. 1, pp. 1-19, 2005. 